\title{
Puertas, ventanas, muros y otros elementos: reflexiones sobre categorías posmodernas contemporáneas
}

\author{
Nuria Casais Pérez \\ Aarhus School of Architecture \\ Ferrán Grau Valldosera \\ Universitat Politècnica de Catalunya
}

Recibido: 4 de julio del 2018 / Aprobado: 5 de septiembre del 2018

doi: 10.26439/limaq2019.n005.4530

En la primera edición de la Bienal deVenecia, The Present of the Past (1980), se escenificó la supremacía del posmodermismo (historicista) y se escuchó la protesta de Jencks, que reivindicaba la importancia de diferenciar las diversas categorías posmodernas. En la Bienal de Venecia del 2014, Fundamentals, se presentó una investigación (retroactiva) sobre los elementos arquitectónicos y una reflexión a nivel global sobre la absorción de la modernidad. Fundamentals se desarrolló a la par que ciertas prácticas de carácter independiente, de dificil clasificación según las categorías establecidas por Jencks en sus árboles genealógicos. Sobre la base de la definición fragmentada (mitad moderno, mitad otra $\cos a)^{\star}$ acuñada por Jencks, se analizan nuevas composiciones posmodernas contemporáneas y se identifican nuevas categorías. En consecuencia,la cuestión por debatir es esta: ¿existe realmente una arquitectura contemporánea a la cual nos podamos referir con el término posmodernismo?

lenguaje, elemento, fragmento, categoría, posmodernismo

\section{Doors, windows, walls and other elements: reflections on contemporary postmodern categories}

In the first Venice Architecture Biennale held in 1980, The Present of the Past, the supremacy of (historicist) postmodernism was reproduced, and Charles Jencks's voice of protest was heard claiming for the importance of distinguishing the different postmodern categories. Years later, in the Venice Architecture Biennale of 2014, Fundamentals, a (retroactive) research on the architectural elements and a global reflection on the absorption of modernity were presented. Fundamentals took place at the same time as certain independent practices, which were difficult to classify according to the categories established by Jencks in his family trees. Based on the fragmented definition (half modern, half something else), new contemporary postmodern compositions are analyzed and new categories are identified. In consequence, the matter of debate is the following: Does a contemporary architecture that could be referred as postmodernism really exist?

language, element, fragment, category, postmodernism

* La frase dos mitades hace referencia al título del libro de Martin Beck (2003). 


\section{DEFINICIÓN FRAGMENTADA DE LA ARQUITECTURA POSMODERNA}

La investigación desarrollada por Charles Jencks (1986), con el objetivo de comprender y divulgar el lenguaje de la arquitectura posmoderna, abordó de nuevo la complejidad y las contradicciones de la disciplina arquitectónica. La elaboración de las siete ediciones de El lenguaje de la arquitectura posmoderna, iniciada durante la década de los setenta, se dilató en el tiempo hasta llegar al año 2002. Las siete ediciones buscaban precisar sucesivamente la mutabilidad de los significados del lenguaje arquitectónico posmoderno y evidenciar la diversidad de sus manifestaciones formales.

Jencks enfocó su trabajo empleando una óptica marcadamente norteamericana, crítica y rigurosa, y haciendo uso de un cierto sentido del humor e ironía propios de la cultura pop. Fue a partir del derribo de algunos bloques del proyecto Pruitt-Igoe — situados en St. Louis (Missouri) - el 15 de julio de 1972 que Jencks declaró la muerte de la arquitectura moderna. Años más tarde, en Nueva Orleans, la Piazza Italia (1975-1979) hizo realidad una de las primeras categorías posmodernas. Durante su investigación, Jencks gestionó las referencias históricas sin prejuicios, con frescura, recordando el tono de Robert Venturi en Complejidad y contradicción en arquitectura. La investigación de Jencks no se limitó al estudio del contexto norteamericano, sino que fue más allá e incluyó ejemplos arquitectónicos clave en Europa o Australia, y algunos referentes relevantes localizados en Japón.

Jencks reivindicó la diversidad de categorías posmodernas, especialmente a propósito de la Bienal de Venecia, The Presence of the Past, al precisar que "el verdadero tema de la bienal era la arquitectura posmoderna" a pesar de cómo se la denominó (1986, p. 156). El título, desafortunadamente, deformó el significado de posmoderno inclinándolo hacia una referencia histórica. Pero la gran virtud de la muestra fue festejar la arquitectura y el nuevo consenso.

Las tentativas de Jencks por clasificar todos los matices del lenguaje posmoderno se tradujeron en sucesivos árboles genealógicos (realmente vivos y evolutivos) que dibujaban paisajes ${ }^{1}$ en los que se relacionaban

1 Charles Jencks ha compatibilizado la teoría y la historia de la arquitectura con la práctica profesional centrada en proyectos de paisajismo. En este sentido, se considera apropiado establecer una vinculación entre la apariencia de sus árboles genealógicos y su práctica profesional. 
categorías arquitectónicas y arquitectos. Estos paisajes contenían historia y presente, y se convirtieron en un documento de referencia que relataba una época de cambio. Durante su evolución, adquirieron incluso mayor complejidad, al punto de necesitar de una representación tridimensional, tal y como recoge The diagrams of architecture (Garcia, 2010). La influencia que estos diagramas han tenido en la disciplina arquitectónica ha sido patente al dar lugar a diferentes adaptaciones e interpretaciones. Rem Koolhaas los ha tomado como referentes en sus trabajos y los ha adaptado desde un punto de vista teórico y práctico. Más recientemente, Alejandro Zaera Polo (2016) ha propuesto una nueva adaptación con el fin de representar la categorización de una serie de prácticas arquitectónicas emergentes a nivel global.

Ante la aparición de nuevas categorías posmodernas, sería lícito cuestionarse si en la actualidad aún existe una arquitectura contemporánea a la cual nos podamos referir con el término posmoderno. Sería precisamente el mismo Charles Jencks quien podría responder a esta pregunta, ya que en las conclusiones de su trabajo propone una definición fragmentada y atemporal de la arquitectura posmoderna, que responde a la siguiente fórmula: "la arquitectura posmoderna es mitad moderna, mitad otra cosa" (Beck, 2003).

En este sentido, sería difícil definir cualquier arquitectura contemporánea sin recurrir a la fórmula propuesta por Jencks, ya que, independientemente de los porcentajes, siempre sería posible encontrar una composición híbrida con base en referentes modernos y otros "no modernos". Por lo tanto, si analizamos la composición de las categorías posmodernas contemporáneas, concluiremos que las categorías previas recogidas en las sucesivas ediciones de El lenguaje de la arquitectura posmoderna - historicismo, los principios del movimiento posmoderno; revivalismo directo; neovernáculo; adhocismo + urbanista $=$ contextualismo; metáfora y metafisica; espacio posmoderno; ¿un eclecticismo radical?, o arquitectura fractal (Jencks, 1986; Beck, 2003)— no incluyen parámetros contemporáneos como, por ejemplo, las problemáticas políticas y sociales aplicadas a la arquitectura, las nuevas tipologías de vivienda, los procesos participativos o los avances de una nueva materialidad y las nuevas técnicas constructivas.

En este sentido, la edición XIV de la Bienal de Venecia (Fundamentals), dirigida por Rem Koolhaas y celebrada treinta y cuatro años después de 
The Presence of the Past, ya recoge los nuevos parámetros contemporáneos al mostrar el estudio de los fundamentos de la arquitectura y el análisis del grado real de absorción de la modernidad. Koolhaas enlaza con la Bienal de 1980 al valerse de la presencia del pasado para redactar manifiestos retroactivos que permiten hacer avanzar la arquitectura. Curiosamente, The Presence of the Past coincidió con la primera participación de Rem Koolhaas en la Bienal de Venecia — probablemente sin imaginar que él un día sería el curador principal一.

Fundamentals (figura 1) señala la relevancia contemporánea del elemento arquitectónico y, en consecuencia, del fragmento arquitectónico, con lo que reafirma la fórmula de Jencks. Intrínsecamente, se subraya también la transcendencia de la capacidad relacional en un proyecto de arquitectura (Absorbing Modernity 1914-2014) (Koolhaas, 2014). Esta edición de la bienal sintonizó, especialmente en el contexto europeo, con prácticas arquitectónicas críticas e independientes, en las que la autonomía de significado del fragmento es compatible con el significado global del proyecto (figura 2).

En consecuencia, esta investigación sobre la herencia del posmodernismo en la arquitectura contemporánea amplía y explica la aparición de nuevas categorías "mitad modernas, mitad otra cosa" atendiendo a la relación que se establece entre los elementos arquitectónicos y entre los diferentes fragmentos que constituyen el proyecto contemporáneo. 
Figura 1. 2014. Pequeña parte de la sección ventanas del archivo de Charles Brooking: "No es la sección de ventanas y guillotinas de un idiota reunida sin una finalidad. Es una fuente útil”.

Fuente: Koolhaas, 2014, p. 212
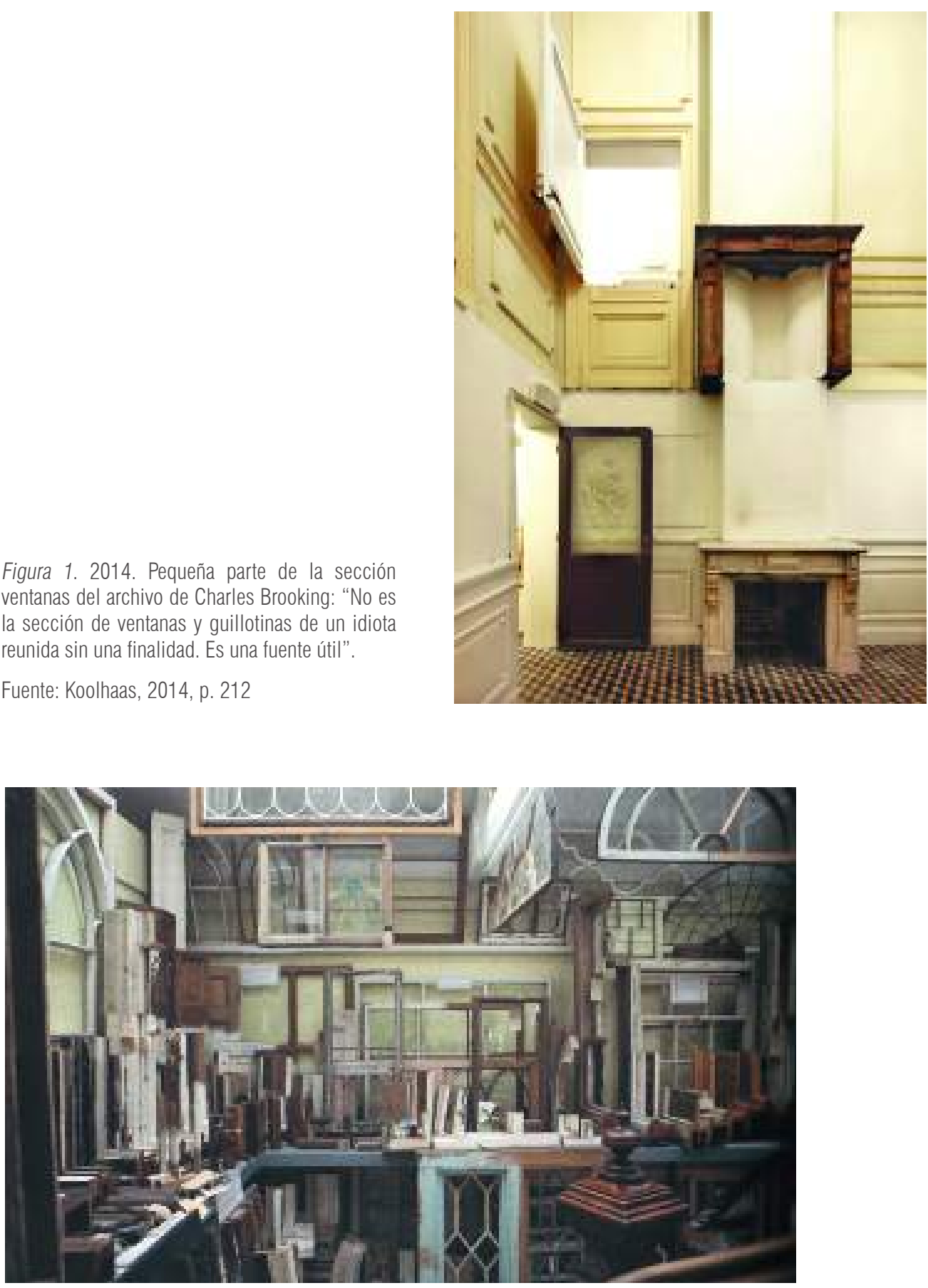

Figura 2. Tienda Twiggy. Proyecto de De Vylder Vinck Taillieu. Vista del espacio central de la tienda, correspondiente a la planta el sótano y la planta baja.

Fuente: De Vylder Vinck Taillieu, 2017, p. 133 


\section{NUEVAS CATEGORÍAS POSMODERNAS}

Situados en el contexto contemporáneo, ¿cuáles serían las nuevas categorías posmodernas y en qué se diferencian de aquellas que definió Charles Jencks? Para responder a esta cuestión analizamos una serie de prácticas arquitectónicas especialmente emplazadas en el contexto europeo, debido a que fue en este continente, en la década de 1980, donde surgieron algunas de las reacciones más críticas y propositivas ante el avance del posmodernismo (historicista). Estas reacciones pretendían contrarrestar aquellas adaptaciones poco informadas que Jencks denomina una "forma degradada del posmodernismo" (Beck, 2003, p. 74). Estas adaptaciones se podrían equiparar a aquellas interpretaciones automáticas y poco reflexivas que se hicieron del estilo internacional, y que desdibujaron su propósito original.

El estudio de algunas prácticas profesionales actuales concluye en cinco categorías posmodernas (contemporáneas): extra-ordinario, ecléctico-social, noble-ornamental, tipológico-doméstico y meta-posmodernismo. Todas ellas contienen componentes en la mitad "otra cosa", que son propios de la contemporaneidad y que ayudan a definir nuevas categorías posmodernas realmente diferenciales. Es importante señalar que estas prácticas arquitectónicas analizadas están vinculadas a prácticas docentes desarrolladas en universidades de referencia, y que, por tanto, son motivo de estudio por la reflexión teórica y práctica que proponen ${ }^{2}$. En consecuencia, estas prácticas se reconocen como referencia y promueven nuevas adaptaciones posmodernas encaminadas a enriquecer el debate arquitectónico.

\section{Extra-ordinario}

La práctica profesional de Anne Lacaton y Jean-Philippe Vassal es el resultado de una actitud comprometida con la disciplina arquitectónica y con la sociedad en la que viven. Una actitud que puede ser calificada como radical y que, tomando prestados términos de los propios arquitectos, valora

2 Anna Lacaton y Jean-Philppe Vassal han sido profesores invitados en Harvard; Tony Fretton ha sido profesor, por ejemplo, en TU Delft y ETH Zurich; Adam Caruso es profesor en la ETH Zurich; Yosiharu Tsukamoto es profesor en el Tokyo Tech, y Jan de Vylder es profesor también en la ETH Zurich. 
la condición extra-ordinaria. Lacaton \& Vassal se preocupa por la calidad del habitar y sus derivas sociales, y no rehúye los valores de lo ordinario. A su vez, asume que la construcción de la arquitectura puede aprovechar procesos industrializados probados en el ámbito de la agricultura, con el objetivo de optimizar la economía y los espacios del proyecto (extra-ordinario), y cumplir con los requerimientos medioambientales actuales. Estos argumentos son los que configuran la "otra cosa", la segunda mitad de la definición de Jencks, y que contribuyen a la revisión crítica de los enunciados de la arquitectura moderna. En términos de lenguaje arquitectónico, este posmodernismo es realmente innovador al proponer una apariencia arquitectónica que no evoca ningún referente anterior y que incluso es interpretado por su crudeza como una no-arquitectura.

La arquitectura de carácter independiente desarrollada en las últimas dos décadas en el contexto británico merece especial atención por su capacidad crítica y la voluntad de proponer alternativas a corrientes comerciales sin muchos compromisos con la disciplina arquitectónica. Estas prácticas están ejerciendo actualmente una influencia notable en el panorama arquitectónico, tanto en el contexto profesional como en el académico, lo que promueve una arquitectura crítica de calidad. Los arquitectos que las representan parten de una revisión y adaptación atenta de los referentes premodernos y comparten una fuerte vinculación con el ámbito del arte contemporáneo (colaboran con artistas, diseñan sus estudios o bien proyectan galerías o museos).

\section{Ecléctico-social}

El arquitecto Tony Fretton adopta, en su práctica profesional, diferentes estilos (clásico, renacentista, moderno) que depura gracias al conocimiento histórico que tiene de ellos, y los combina con una actitud social y cultural comprometida, especialmente en la definición de equipamientos públicos, como la Lisson Gallery. Con este proyecto reivindicó, durante el inicio de su carrera, la democratización de la cultura durante el mandato de Margaret Tatcher. El posmodernismo ecléctico-social recupera en las propuestas de vivienda los fundamentos básicos del Movimiento Moderno y ofrece mejoras en las condiciones de habitabilidad. El diseño de la Red House (Tony 
Fretton Architects, 2001), situada en el barrio de Chelsea en Londres, define ejemplarmente las condiciones de habitabilidad recurriendo al valor de cada uno de los referentes históricos utilizados. A pesar de la apariencia unitaria de esta vivienda, conseguida gracias a un revestimiento continuo de piedra roja y la abstracción elegante de los elementos característicos de las viviendas vecinas (basamento, galería, línea de cornisa, la arquitectura de la cubierta, etcétera), la Red House es un proyecto absolutamente fragmentado. Un proyecto construido a manera de estratos, en el que cada planta corresponde a un estilo diferente. La planta baja es moderna, la primera planta es renacentista, la entreplanta es racionalista y el ático, moderno de nuevo. Esta fragmentación solo es perceptible desde la experiencia interior y tiene la capacidad de no repercutir en absoluto en la coherencia global del proyecto.

Figura 3. Fachada principal de la Red House, de Tony Fretton

Fuente: Tony Fretton Architects, 2001, p. 121

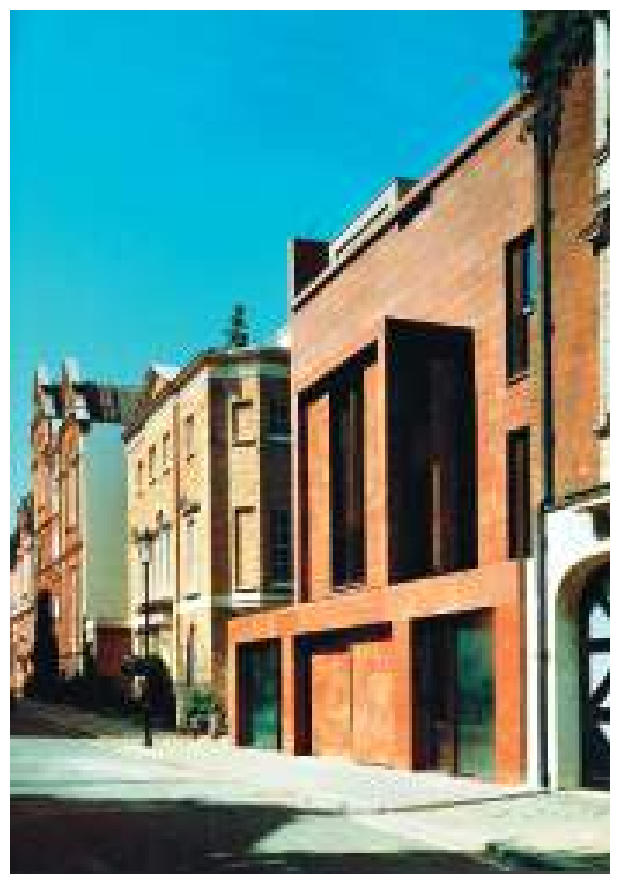




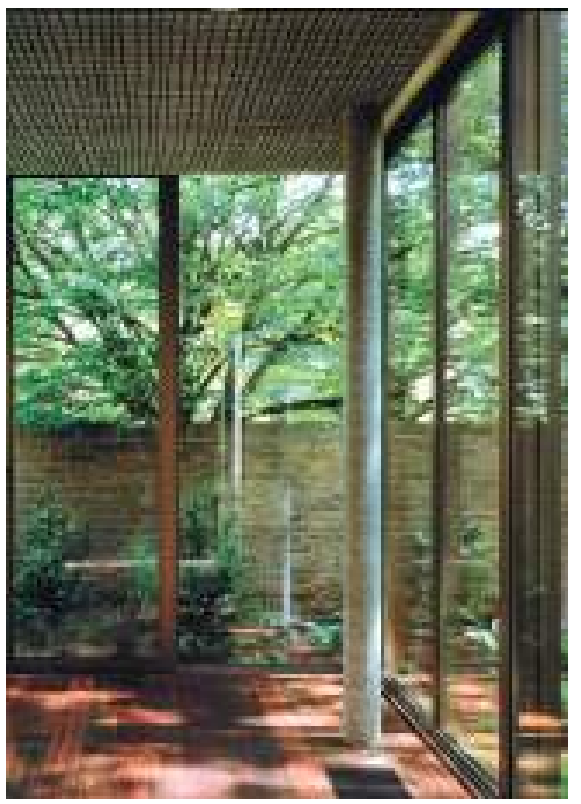

Figura 4. Planta baja de la Red House

Fuente: Tony Fretton Architects, 2001, p. 128

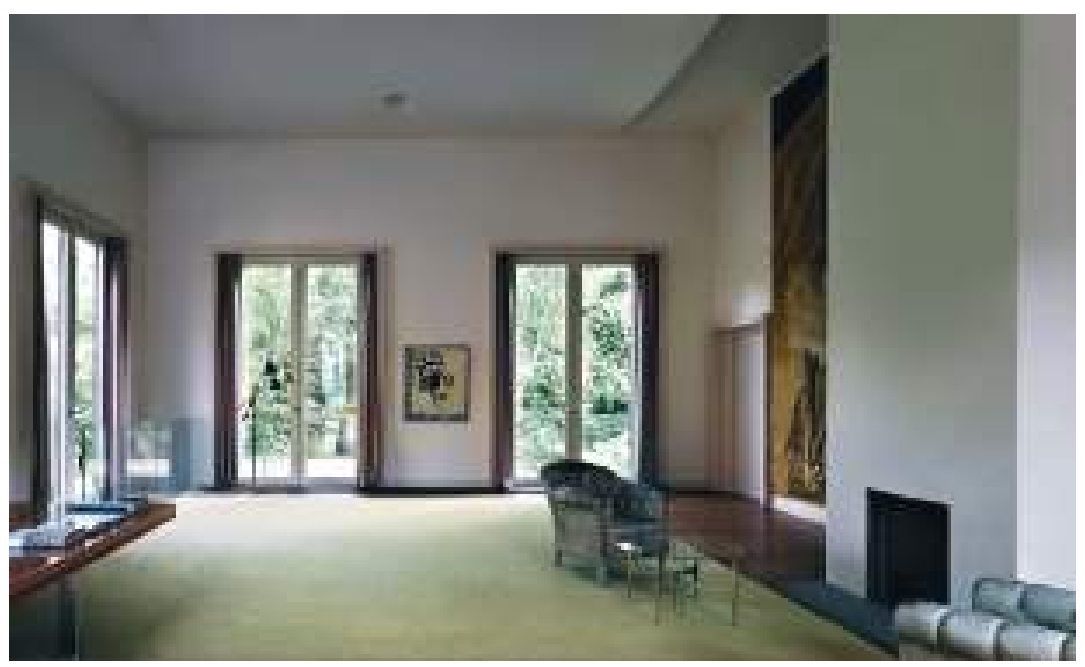

Figura 5. Primera planta de la Red House

Fuente: Tony Fretton Architects, s. f. 


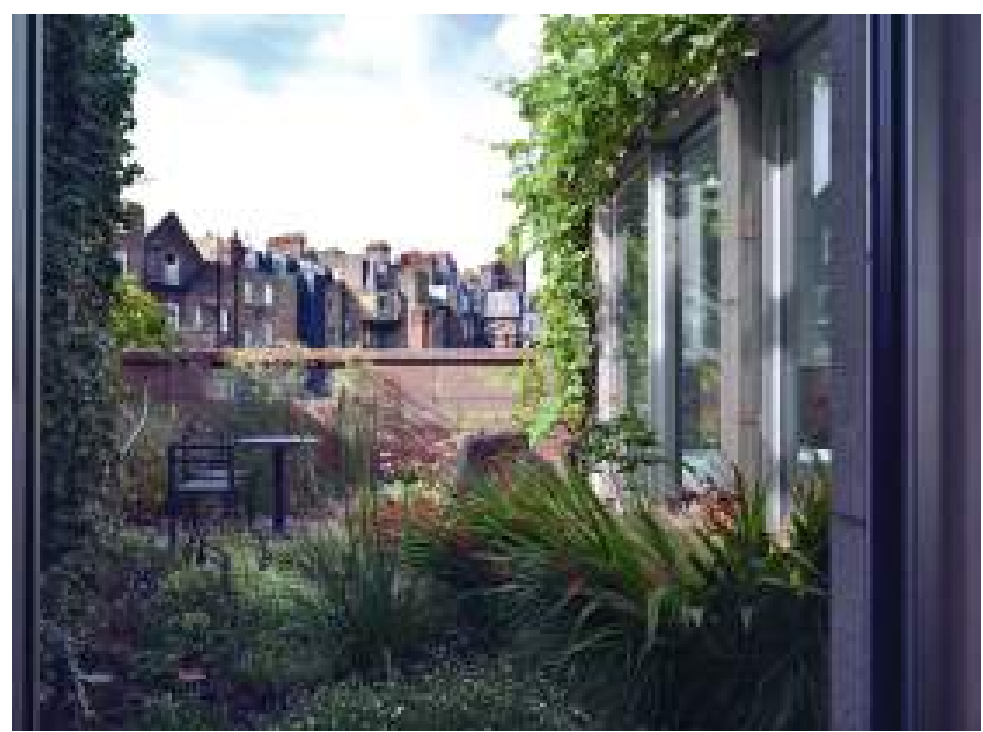

Figura 6. Ático de la Red House

Fuente: Tony Fretton Architects, s. f.

\section{Noble-ornamental}

En la actualidad, el estudio Caruso \& St. John profesa una devoción por la tradición arquitectónica, especialmente aquella vinculada a la época medieval. Esta práctica muestra un gran interés por la capacidad de la ornamentación esencial para dotar a la arquitectura de un aura próxima y reconocible para el usuario. Es notable también la admiración que profesan por la esencialidad del trabajo de Sigurd Lewerentz, tanto en su primera etapa clásica como en su madurez moderna, y que se hace evidente en el proyecto de la Brick House (2005). Caruso \& St. John son herederos reconocidos del clasicismo de Hans Kollhoff y apuestan por una arquitectura de apariencia noble que a veces roza lo monumental. $\mathrm{Al}$ mismo tiempo, sus proyectos prestan mucha atención a la condición cotidiana y humana de la arquitectura (véase la revista El Croquis 187). Es precisamente esta condición de proximidad la que les empuja a proponer una arquitectura muy personal, casi artística. En sus proyectos, a diferencia del posmodernismo historicista, las costuras 
entre la parte moderna y la otra mitad son casi imperceptibles, y en ciertos momentos resulta dificil detectar si la arquitectura que experimentamos fue construida en la actualidad o antaño.

\section{Tipológico-doméstico}

La práctica del estudio Atelier Bow-Wow, liderado por Yoshiharu Tsukamoto y Momoyo Kaijima, debe ser entendida a partir de su fuerte compromiso con la docencia, la investigación, el arte contemporáneo y la divulgación de su trabajo. Aunque básicamente su práctica y docencia se desarrollan en Japón (país referente también en la investigación de Jencks), la cultura europea es parte esencial de su formación. El trabajo de Atelier Bow-Wow se centra a menudo en el estudio de tipologías históricas y contemporáneas, $\mathrm{y}$ atiende también a las condiciones sociales que influyen verdaderamente en la arquitectura, entendidas aquí como comportamiento (Atelier Bow-Wow, 2010). La combinación de ambos intereses da lugar, en términos de lenguaje arquitectónico, a propuestas absolutamente críticas, libres e independientes. La práctica de Atelier Bow-Wow sugiere que una nueva categoría posmoderna podría denominarse tipológica-doméstica. Los proyectos de sus viviendas unifamiliares con un sistema de representación integral, sus famosas secciones fugadas (Atelier Bow-Wow, 2008) son un referente y un claro ejemplo de esta estrategia proyectual contemporánea. El reconocimiento exhaustivo de la realidad, afrontado como una investigación (Atelier Bow-Wow, 2001, 2002), promueve un diseño arquitectónico aparentemente anónimo en el que la autoría queda en segundo plano, detrás del resultado final.

\section{Meta-posmodernismo}

La última categoría que se evidencia en esta investigación es la que deriva de la práctica del estudio belga De Vylder Vinck Taillieu (ADVVT). En cierto modo, esta categoría reúne en la mitad "otra cosa" (la complementaria a la mitad moderna) parámetros e intereses de las cuatro categorías abordadas antes. En este sentido, se reconoce una arquitectura en ciertos momentos tan "inacabada" y tan crítica como la del posmodernismo extra-ordinario; una habitabilidad verdaderamente al servicio del usuario y un eclecticismo extremo y comprometido afin al posmodernismo ecléctico-social; una devoción por el 
ornamento al servicio de las escenas cotidianas similar a la del posmodernismo noble-ornamental, y una investigación tipológica afin a la construcción de la domesticidad propia del posmodernismo tipológico-doméstico.

La categoría posmoderna que deriva del estudio ADVVT da un paso más allá e hibrida de una forma sorprendente el Movimiento Moderno, al que actualiza. Ello da lugar a lo que podríamos denominar meta-posmodernismo, en el sentido de 'después del posmodernismo'. Las propuestas arquitectónicas de ADVVT suelen ser el resultado de la adición y combinación de diferentes fragmentos. Estos fragmentos corresponden, en muchas ocasiones, a estilos arquitectónicos distintos o a sus combinaciones. Esta estrategia se repite tanto si se trata de un proyecto de rehabilitación (la mayoría de los casos) como de un proyecto de nueva planta.

La complejidad aumenta especialmente cuando ADVVT recurre a fragmentos construidos con diferentes combinaciones de estilos arquitectónicos. Cada fragmento contiene elementos arquitectónicos de origen diverso que toman un nuevo sentido precisamente por la propuesta relacional de los arquitectos. Esta capacidad relacional es la que se exhibe en Bravoure Scarcity Beauty ${ }^{3}$ y que aporta nuevas claves respecto al proyecto de Fundamentals (figuras 7 y 9). Bravoure Scarcity Beauty "mostró un conjunto de proyectos de varios estudios flamencos que se explican a través de réplicas de tamaño natural de un detalle significativo" (Quintáns, 2018, p. 318). En la exposición se subrayaba la capacidad relacional entre los elementos de todos aquellos proyectos y entre los fragmentos de un mismo proyecto.

La manera en que se gestiona la relación entre estos elementos en el trabajo de ADVVT evoca la estrategia posmoderna del historicismo o del revivalismo directo (Jencks, 1986, pp. 81-90). Pero en este caso, el ejercicio consiste en "posmodernizar" la arquitectura empleando de otro modo los elementos que definieron antaño el estilo internacional (el pilar, la ventana corrida, etcétera) y en proponer nuevas versiones de los componentes fundamentales de la arquitectura (muros cortina con rotura de puente térmico, o plásticos o tejidos de última generación).

3 Proyecto curatorial de ADVVT conjuntamente con Doorzon Interior Architects y Filip Dujardin para la Bienal de Venecia 2016. 
En el meta-posmodernismo es habitual cortar los edificios, a la manera de Gordon Matta-Clark, y generar ciertos desplazamientos que alteran una realidad común; gracias a dicha deformación, esta realidad adquiere una condición absolutamente singular (figura 8).

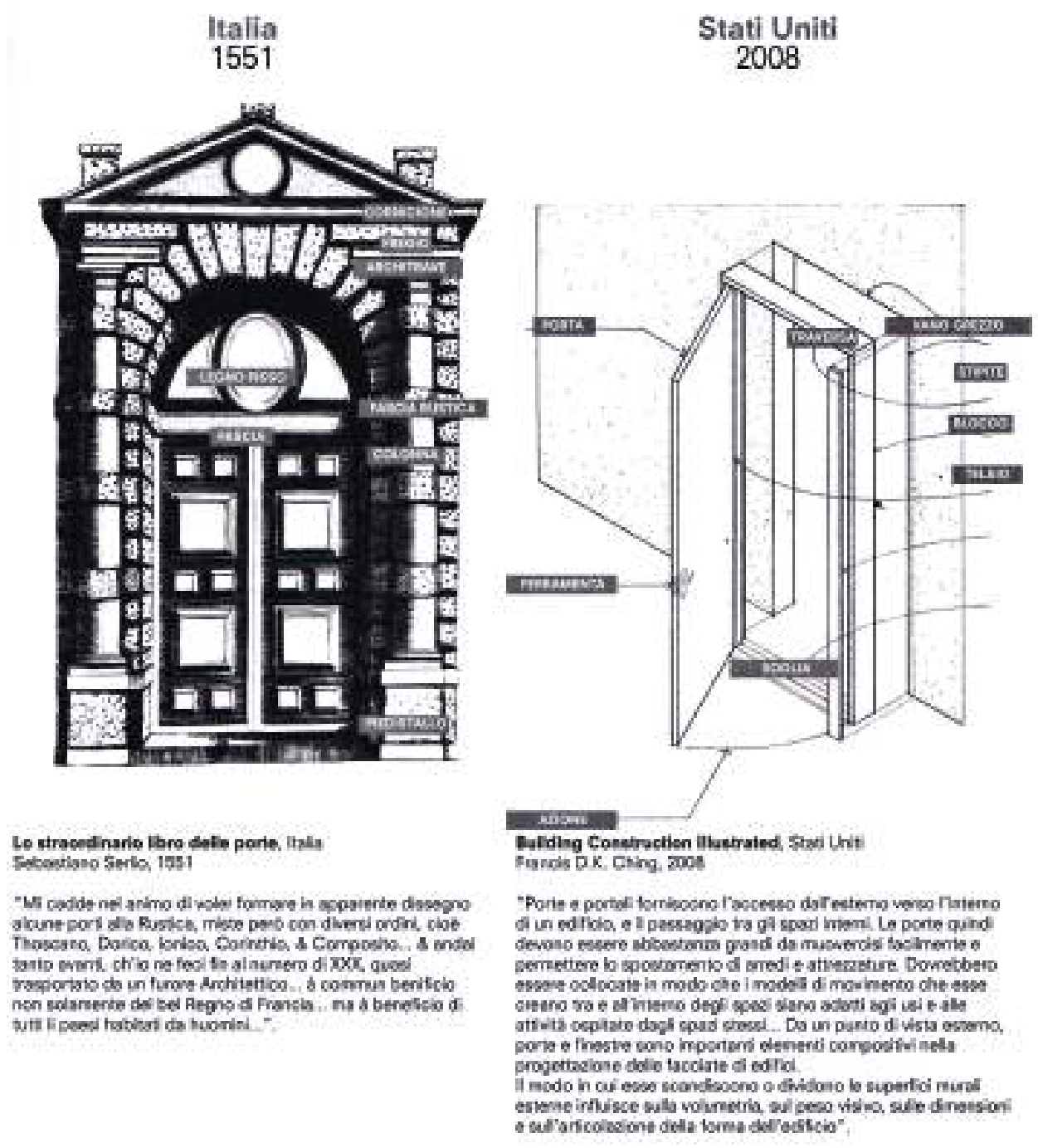

Figura 7. Estudio histórico sobre tipologías de puertas

Fuente: Koolhaas, 2014, p. 258 


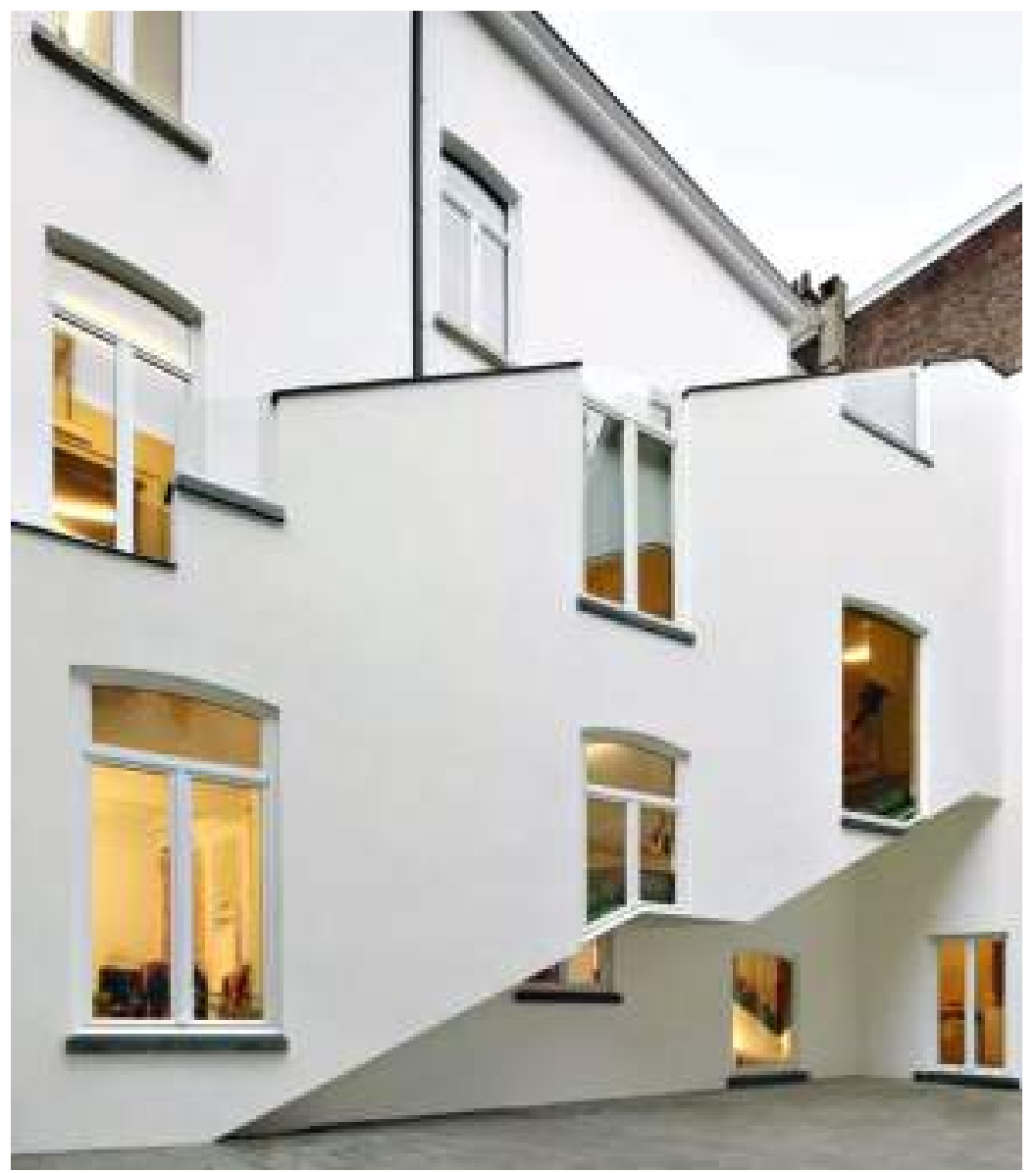

Figura 8. Tienda Twiggy, proyecto de De Vylder Vinck Taillieu. Vista desde el patio que evidencia los desplazamientos del plano de la fachada

Fuente: De Vylder Vinck Taillieu, 2017, p. 129

En otros proyectos, la estrategia consiste en desacreditar la materialidad. En este sentido, algunos edificios presentan muros de ladrillo que en realidad son puertas secretas (casa Weze), muros aparentemente de fachada que esconden un patio exterior (casa Verbrande Brug) (figura 10); montantes 
que usualmente quedan ocultos (balloon frame) y puntales de obra que permanecen se convierten en un acabado en apariencia inconcluso (casa Rot Ellen Berg). También entienden como lícita la superposición de un muro cortina absolutamente transparente delante de un muro de ladrillo, así como la superposición de tendales domésticos sobre la superficie lisa y reflectante del citado muro cortina (Les Ballets $\mathrm{C}$ de la B and LOD).

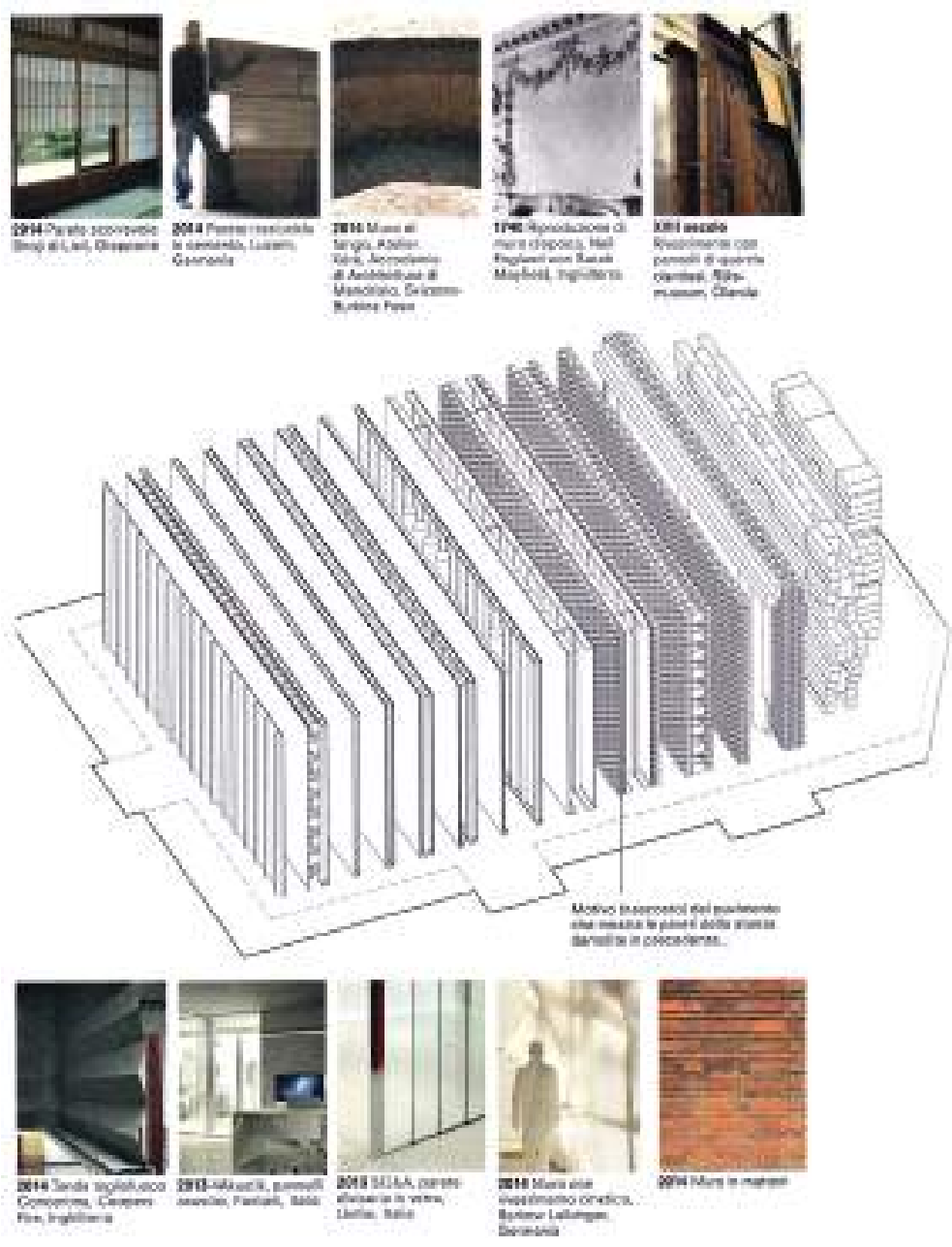

Figura 9. Estudio histórico sobre tipologías de muros

Fuente: Koolhaas, 2014, p. 266 


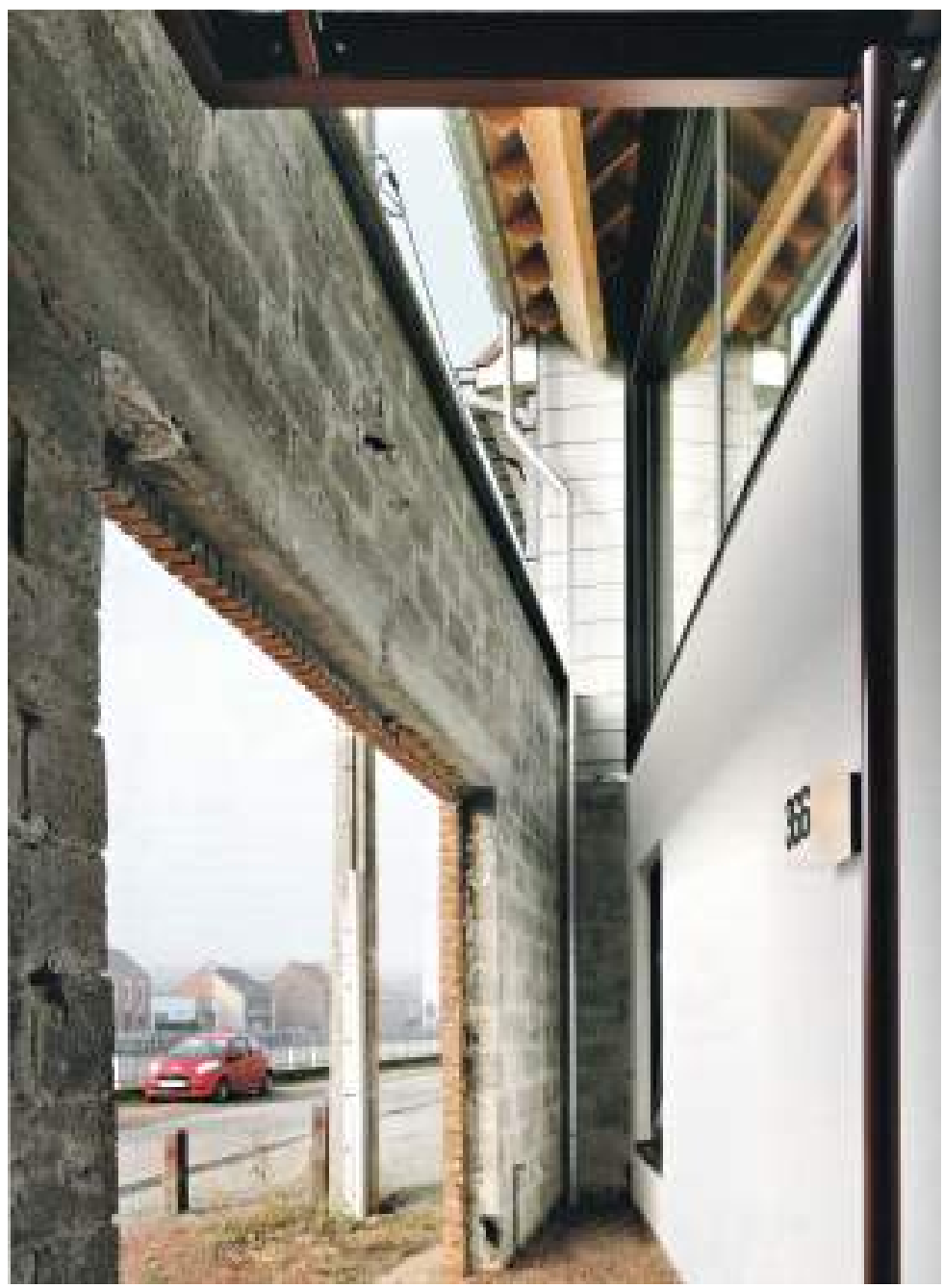

Figura 10. Casa Verbrande Brug, proyecto de De Vylder Vinck Taillieu. Espacio intersticial entre las paredes del antiguo garaje y la fachada de la nueva casa

Fuente: De Vylder Vinck Taillieu, 2017, p. 26 


\section{CONCLUSIONES}

Una visión global sobre las categorías posmodernas contemporáneas expuestas anteriormente constata el hecho de que cada una de ellas está asociada a una nacionalidad y a un contexto local concretos. En este sentido, esta situación evoca los fundamentos del regionalismo crítico (Frampton, 1985) y evidencia que, ante la deriva de las categorías posmodernas acuñadas inicialmente por Jencks, la arquitectura de escala pequeña (fruto de las consecuencias de una crisis económica a escala global) fomenta la crítica y la reflexión. Este contexto de proximidad, en el que las condiciones del proyecto se conocen en profundidad, posibilita el avance de la disciplina y proyecta sus resultados a nivel global. También es trascendente que estas categorías se desarrollen paralelamente en el contexto académico, pues consolidan la solvencia intelectual de estas prácticas.

El análisis de estas arquitecturas contemporáneas demuestra que la fórmula propuesta por Jencks para definir el posmodernismo "mitad moderno-mitad otra cosa" (Beck, 2003, p. 67) es aún vigente, a pesar de que requiere la incorporación de nuevas categorías. Por otro lado, esta investigación también reafirma el valor de la multivalencia frente a la univalencia (utilizando términos de Jencks) y corrobora a su vez el valor de la complejidad y la capacidad de relacionar elementos y fragmentos. En definitiva, De Vylder Vinck Taillieu y Jencks coinciden al incidir en el valor de la capacidad relacional. ADVVT constata que "cosas que no tienen nada que ver unas con otras, de repente pasan todas a tener algo que ver entre ellas" (De Vylder Vinck Taillieu, 2017, p. 162), mientras que Jencks afirma que "la noción de interrelaciones entre cosas deviene tan importante como las cosas por sí mismas" (Beck, 2003, p. 67). Estas cosas son puertas, ventanas, muros y otros elementos, y también todas aquellas condiciones no visibles que las relaciona. 


\section{REFERENCIAS}

Atelier Bow-Wow. (2001). Made in Tokyo: Guide book. Japón: Kajima Institute Publishing.

Atelier Bow-Wow. (2002). Pet architecture guide book. Japón:World Photo.

Atelier Bow-Wow. (2008). Graphic anatomy:Atelier Bow-Wow. Japón:Toto.

Atelier Bow-Wow. (2010). The architectures of Atelier Bow-Wow: Behaviorology. Nueva York: Rizzoli.

Beck, M. (2003). Half modern, half something else (Charles Jencks, "The language of post-modern architecture", first, second, third, fourth, fifth, sixth, and seventh editions). Nueva York, Viena: Florian Pumhöl.

De VylderVinck Taillieu. (2017). Architecture is not a matter of architecture. $A+U,(561), 162$.

Frampton, K. (1985). Historia crítica de la arquitectura moderna (4. ${ }^{a}$ ed.). Barcelona: Gustavo Gili.

Garcia, M. (2010). The diagrams of architecture. Chichester: Wiley.

Jencks, C. (1986). El lenguaje de la arquitectura posmoderna (3. ${ }^{a}$ ed). Barcelona: Gustavo Gili.

Koolhaas, R. (2014). Fundamentals.Venice: Marsilio.

Quintáns, C. (mayo del 2018). Optimismo. Archives. Journal of Architecture [Architecten De VylderVinck Taillieu], (3), 314-322.

Tony Fretton Architects. (2001). A+T, (18), 118-149.

Tony Fretton Architects. (s. f.). The Red House, London, UK. Recuperado de http://www.tonyfretton.com/redhouse/index.html

Zaera Polo,A. (2016).Ya bien entrado el siglo XXI, ¿las arquitecturas del postcaptialismo? El Croquis [Sergison Bates 2004-2016], (187), 252-287. 